\title{
Assessing the tension between a black hole dominated early universe and leptogenesis
}

\author{
Yuber F. Perez-Gonzalez $\circledast^{1,2,3, *}$ and Jessica Turner $\circledast^{4, \dagger}$ \\ ${ }^{1}$ Theoretical Physics Department, Fermi National Accelerator Laboratory, \\ P.O. Box 500, Batavia, Illinois 60510, USA \\ ${ }^{2}$ Department of Physics \& Astronomy, Northwestern University, Evanston, Illinois 60208, USA \\ ${ }^{3}$ Colegio de Fisica Fundamental e Interdisciplinaria de las Americas (COFI), \\ 254 Norzagaray street, San Juan 00901, Puerto Rico \\ ${ }^{4}$ Institute for Particle Physics Phenomenology, Department of Physics, Durham University, \\ South Road, Durham DH1 3LE, United Kingdom.
}

(Received 5 November 2020; revised 23 February 2021; accepted 21 October 2021; published 16 November 2021)

\begin{abstract}
We perform the first numerical calculation of the interplay between thermal and black hole induced leptogenesis, demonstrating that the right-handed neutrino surplus produced during the evaporation only partially mitigates the entropy dilution suffered by the thermal component. As such, the intermediate-mass regime of the right-handed neutrinos, $10^{6} \mathrm{GeV} \lesssim M_{N} \lesssim 10^{9} \mathrm{GeV}$, could not explain the observed baryon asymmetry even for fine-tuned scenarios if there existed a primordial black hole dominated era, consistent with initial black hole masses of $M_{i} \gtrsim \mathcal{O}(1) \mathrm{kg}$ and initial energy density fractions larger than $\beta^{\prime} \gtrsim 10^{-6}$. A possible detection of the gravitational waves emitted from the same primordial black holes would place intermediate-scale thermal leptogenesis under tension. https://github.com/earlyuniverse/ulysses
\end{abstract}

DOI: $10.1103 /$ PhysRevD.104.103021

\section{INTRODUCTION}

Primordial black holes (PBHs) could have been formed after the big bang through many different mechanisms [1-3]. Once created, PBHs constitute an increasingly large portion of the universe's energy budget playing an essential role in the universe's evolution. Hawking's seminal papers $[4,5]$ led to his discovery that black holes evaporate over time. The fate of these PBHs is unknown: it is possible they disappear or leave a relic which can constitute a portion of dark matter [6-12]. Nonetheless, during their evaporation, the PBHs will produce all possible particles with masses below its temperature. This democratic feature of PBHs can lead to the production of dark matter [13-15], dark radiation [16-20], and the matter-antimatter asymmetry. The latter idea was initially explored in [4,21-23] where heavy, new particles, produced from PBH evaporation, can decay in a $C P$ - and baryon-number- violating manner to produce the observed baryon asymmetry [24,25].

\footnotetext{
yfperezg@northwestern.edu

jessica.turner@durham.ac.uk
}

Published by the American Physical Society under the terms of the Creative Commons Attribution 4.0 International license. Further distribution of this work must maintain attribution to the author(s) and the published article's title, journal citation, and DOI. Funded by SCOAP ${ }^{3}$.
Several works have examined the connection between neutrino masses, the matter-antimatter asymmetry and PBHs, in scenarios where dark matter and right-handed $(\mathrm{RH})$ neutrinos are produced via Hawking radiation $[13,14]$. The nonthermal decays of the RH neutrinos violate $C P$ and lepton number, resulting in the generation of a lepton asymmetry converted to a net excess of baryons via nonperturbative Standard Model effects [26]. This process, known as leptogenesis, links the origin of light neutrino masses, suppressed by the heavy, RH neutrino mass, with the baryon asymmetry [27-31].

In this article, we compute, for the first time, the numerical solutions to the Friedmann equations for the evolution of a $\mathrm{PBH}$ dominated universe and the baryon asymmetry produced from thermal leptogenesis together with the nonthermal contribution generated by the $\mathrm{PBH}$ evaporation. We show that the baryon asymmetry undergoes a dilution due to the entropy injection from the black hole evaporation. We quantify such depletion in the intermediate-scale regime, $10^{6} \lesssim M_{N}(\mathrm{GeV}) \lesssim 10^{9}$, and find that even for the most optimistic scenarios in this regime, the baryon asymmetry would be insufficient to explain the observed value for initial $\mathrm{PBH}$ masses $M_{i} \gtrsim \mathcal{O}(1) \mathrm{kg}$ and $\mathrm{PBH}$ energy density fractions to the total density at formation $\beta^{\prime} \gtrsim 10^{-6}$. Finally, we discuss the gravitational wave spectrum and highlight that such signals' future detection would falsify thermal leptogenesis in the intermediate regime. Our code will be made publicly 
available through the ULYSSES PYTHON package [32]. We consider natural units throughout.

\section{TYPE I SEESAW MECHANISM}

The seesaw mechanism [33-40] is a compelling model that simultaneously explains the smallness of neutrino masses and the origin of the matter antimatter asymmetry. In the most minimal framework, the Standard Model (SM) Lagrangian is augmented to include at least two heavy RH neutrinos with masses $M_{N_{i}}$ :

$$
\mathcal{L}=i \overline{N_{i}} \not N_{i}-\overline{L_{\alpha}} Y_{\alpha i} N_{i} \tilde{\Phi}-\frac{1}{2} \overline{N_{i}^{C}} M_{N_{i}} N_{i}+\text { H.c. },
$$

where $N_{i}, L_{\alpha}$ and $\Phi$ denote the RH neutrinos of generation $i, \mathrm{SU}(2)_{L}$ leptonic doublets of flavor $\alpha$ and Higgs doublets, respectively, with the negative hypercharge Higgs doublet defined as $\tilde{\Phi}=i \sigma_{2} \Phi^{*}$. In its original manifestation [27], the RH neutrinos can decay out of thermal equilibrium at temperatures similar to their mass. Further, if the Yukawa matrix, $Y_{\alpha i}$, contains complex phases these decays can produce more antileptons than leptons (or vice versa) and the resultant lepton asymmetry is converted via electroweak sphaleron processes [26] to a baryon asymmetry. This mechanism is commonly known as nonresonant thermal leptogenesis and the minimal mass scale is $M_{N_{i}} \gtrsim 10^{6} \mathrm{GeV}$ [41]. ${ }^{1}$ In the following, we focus on this minimal nonresonant thermal leptogenesis and its interplay with a black hole dominated earlier universe.

The Yukawa matrix can be conveniently parametrized such that neutrino oscillation data is automatically recovered [43],

$$
Y=\frac{1}{v} U \sqrt{m_{\nu}} R^{T} \sqrt{M_{N}},
$$

where $v=174 \mathrm{GeV}, U$ is the leptonic mixing matrix, $m_{\nu}$ is the diagonal light neutrino mass matrix, $R$ is a complex, orthogonal matrix and $M_{N}$ is the diagonal mass matrix of the heavy RH neutrinos. The model parameter space is 18 dimensional where nine parameters are associated with the low-energy scale physics, and the remaining nine parameters are associated with the high-scale physics of the RH neutrinos.

\section{RIGHT-HANDED NEUTRINO PRODUCTION FROM HAWKING RADIATION}

Black holes (BHs) evaporate by emitting a thermal flux of particles, known as Hawking radiation [4,5]. Such a flux is generated because of the gravitational disruption created by the collapsing matter forming the $\mathrm{BH}$ [44]. The emission

\footnotetext{
${ }^{1}$ In this regime, the mass splittings of the $\mathrm{RH}$ neutrinos is order one and therefore far from the resonant regime. This was also explored in [42].
}

is democratic in nature, i.e., all particles existing in the universe can be emitted independently of their interactions. Therefore, if RH neutrinos do indeed exist, they would be produced during the evaporation. The instantaneous emission rate of RH neutrino $N_{i}$, with momentum between $p$ and $p+d p$ and time interval $d t$, is [4,5]

$$
\frac{d^{2} \mathcal{N}_{N_{i}}}{d p d t}=\frac{g_{i}^{N}}{2 \pi^{2}} \frac{\sigma_{\mathrm{abs}}^{1 / 2}\left(M, M_{N_{i}}, p\right)}{\exp \left[E_{i}(p) / T_{\mathrm{BH}}\right]+1} \frac{p^{3}}{E_{i}(p)},
$$

where $g_{i}^{N}$ are the internal degrees-of-freedom (dof) of RH neutrinos, $\sigma_{\text {abs }}^{1 / 2}\left(M, M_{N_{i}}, p\right)$ is the absorption cross section for a massive fermion [45-47], $E_{i}(p)$ the $\mathrm{RH}$ neutrino total energy, and $T_{\mathrm{BH}}$ the instantaneous $\mathrm{BH}$ temperature. In the case of a Schwarzschild $\mathrm{BH}$, its temperature $T_{\mathrm{BH}}$ is related to its mass $M$ as

$$
T_{\mathrm{BH}}=\frac{1}{8 \pi G M} \approx 1.06 \mathrm{GeV}\left(\frac{10^{13} \mathrm{~g}}{M}\right) .
$$

For BH temperatures $T_{\mathrm{BH}} \ll M_{N_{i}}$, we observe that the emission of RH neutrinos becomes highly suppressed. This suppression will impose a constraint on the range of BH masses that will contribute in a significant manner to leptogenesis.

As a result of the evaporation process, $\mathrm{BHs}$ lose mass over time at a rate $[48,49]$,

$$
\begin{aligned}
\frac{d M}{d t} & =-\sum_{a} \frac{g_{a}}{2 \pi^{2}} \int_{0}^{\infty} \frac{\sigma_{\mathrm{abs}}^{s_{a}}(G M p) p^{3} d p}{\exp \left[E_{a}(p) / T_{\mathrm{BH}}\right]-(-1)^{2 s_{a}}}, \\
& =-\kappa \varepsilon(M)\left(\frac{1 \mathrm{~g}}{M}\right)^{2}
\end{aligned}
$$

where the sum is performed over all existing particles with spin $s_{a}$, dofs $g_{a}$ and absorption cross section $\sigma_{\text {abs }}^{s_{a}}(G M p)$. The mass lose rate in Eq. (5) is conveniently parametrized in terms of an evaporation function, $\varepsilon(M)$, normalized to the unity for $M \gg 10^{17} \mathrm{~g}$ where $\kappa=5.34 \times 10^{25} \mathrm{~g} \mathrm{~s}^{-1}$ $[48,49]$. For the case of type-I seesaw framework, the evaporation function has both SM and RH neutrino contributions, $\varepsilon(M)=\varepsilon_{\mathrm{SM}}(M)+\varepsilon_{N}(M)$ [17]:

$$
\varepsilon_{N}(M) \approx 2 n_{N_{i}} f_{1 / 2}^{0} \sum_{i=1}^{n_{N_{i}}} \exp \left[-\frac{8 \pi G M M_{N_{i}}}{4.53}\right],
$$

where $n_{N_{i}}=3$ denotes the number of generations of RH neutrinos, and the factor $f_{1 / 2}^{0}$ corresponds to the neutral fermion contribution to the evaporation function [49]. The presence of the exponential factor characterizes the $\mathrm{BH}$ mass where the RH neutrino emission becomes suppressed. 


\section{THE INTERPLAY BETWEEN LEPTOGENESIS AND EARLY BLACK HOLE DOMINATION}

Let us assume an initial density of PBHs, $\rho_{\mathrm{PBH}}^{i}$, formed after inflation with a monochromatic mass distribution. The initial PBH mass is proportional to the initial radiation density at the formation time, $M_{i} \propto \gamma \rho_{\mathrm{tot}}^{i} H_{i}^{-3}$, where $\gamma$ is a dimensionless gravitational collapse parameter and $H_{i}$ the Hubble parameter [1]. The ratio of the initial $\mathrm{PBH}$ fraction to the total energy, $\rho_{\text {tot }}^{i}$, for a given plasma temperature at the formation $T_{\mathrm{f}}$, is [1]

$$
\beta^{\prime}=\gamma^{1 / 2}\left(\frac{g_{*}\left(T_{\mathrm{f}}\right)}{106.75}\right)^{-1 / 4} \frac{\rho_{\mathrm{PBH}}^{i}}{\rho_{\mathrm{tot}}^{i}}
$$

Depending on the value of $\beta^{\prime}$, the PBHs could eventually dominate the evolution of the universe before their evaporation. We consider PBHs in the mass range $\left(10^{-1} \lesssim M_{i}(\mathrm{~g}) \lesssim\right.$ $10^{4}$ ) where the upper boundary derives from PBH evaporation that would occur before the electroweak phase transition and the lower from constraints on inflation [19,50].

If the type-I seesaw mechanism explains neutrino masses, then RH neutrinos would be produced in the early universe in two ways, by the BHs when their temperature $T_{\mathrm{BH}} \ll M_{N_{1}}$ and by the thermal plasma. To calculate the resultant baryon asymmetry we track the evolution of the comoving number density of the RH neutrinos, PBHs, lepton asymmetry and radiation energy density using momentum integrated Boltzmann equations. These equations assume that kinetic equilibrium of the RH neutrinos and neglect quantum statistics. We find that for the points in the model parameter space we explore, leptogenesis occurs in the strong washout regime where it has been shown that momentum averaged Boltzmann equations yields a near-identical lepton asymmetry compared to the full treatment [51].

We consider the viability of nonresonant thermal leptogenesis and assume a mass splitting of $M_{2}=3.15 M_{1}$ and $M_{3}=3.15 M_{2}$ and $10^{6} \lesssim M_{1}(\mathrm{GeV}) \lesssim 10^{13}$. The upper and lower boundary reflects the viable region for nonresonant thermal. The Friedmann equations for the comoving energy density of radiation $\left(\varrho_{\mathrm{R}}=a^{4} \rho_{\mathrm{R}}\right)$ and $\mathrm{PBHs}\left(\varrho_{\mathrm{BH}}=a^{3} \rho_{\mathrm{BH}}\right)$ with respect to the scale factor $a$ are

$$
\begin{aligned}
\frac{d \varrho_{\mathrm{R}}}{d a} & =\frac{1}{a \Delta}[4(\Delta-1)-\Sigma]-\frac{\varepsilon_{\mathrm{SM}}(M)}{\varepsilon(M)} \frac{1}{M} \frac{d M}{d a} a \varrho_{\mathrm{BH}}, \\
\frac{d \varrho_{\mathrm{BH}}}{d a} & =\frac{1}{M} \frac{d M}{d a} \varrho_{\mathrm{BH}}, \\
H^{2} & =\frac{8 \pi G}{3}\left(\varrho_{\mathrm{BH}} a^{-3}+\varrho_{\mathrm{R}} a^{-4}\right),
\end{aligned}
$$

where $H$ is the Hubble rate. The first term in Eq. (8a) takes into account the change of the effective number of relativistic degrees of freedom $g_{*}(T)$ as the Universe cools down, with $\Sigma$ and $\Delta$ functions defined as

$\Sigma=\frac{T}{g_{*}(T)} \frac{d g_{*}(T)}{d T}, \quad \Delta=1+\frac{T}{3 g_{* S}(T)} \frac{d g_{* S}(T)}{d T}$.

The PBH evaporation implies that the entropy of the universe is not conserved. Therefore, we need to follow the evolution of the ambient temperature, $T$, using $[17,52,53]$

$$
\frac{d T}{d a}=-\frac{T}{\Delta}\left\{\frac{1}{a}+\frac{\varepsilon_{\mathrm{SM}}(M)}{\varepsilon(M)} \frac{1}{M} \frac{d M}{d a} \frac{g_{*}(T)}{g_{* S}(T)} \frac{a \varrho_{\mathrm{BH}}}{4 \varrho_{\mathrm{R}}}\right\} .
$$

We emphasize that the ambient plasma temperature, $T$, is not necessarily the same as the temperature of the black holes, $T_{\mathrm{BH}}$. To address the generation of baryon asymmetry, we require Boltzmann equations for the evolution of the comoving RH neutrino number density and the $B-L$ asymmetry produced from the decays (and washout) of the RH neutrinos. We assume a vanishing initial abundance of RH neutrinos. They are populated thermally, from inverse decays of leptons and Higgses, and nonthermally from the Hawking radiation. For the sake of clarity, let us separate the equations for the thermal $\left(n_{N_{1}}^{\mathrm{TH}}\right)$ and nonthermal $\left(n_{N_{1}}^{\mathrm{BH}}\right)$ densities, ${ }^{2}$

$$
\begin{aligned}
& a H \frac{d n_{N_{1}}^{\mathrm{TH}}}{d a}=-\left(n_{N_{1}}^{\mathrm{TH}}-n_{N_{1}}^{\mathrm{eq}}\right) \Gamma_{N_{1}}^{T}, \\
& a H \frac{d n_{N_{1}}^{\mathrm{BH}}}{d a}=-n_{N_{1}}^{\mathrm{BH}} \Gamma_{N_{1}}^{\mathrm{BH}}+n_{\mathrm{BH}} \Gamma_{\mathrm{BH} \rightarrow N_{1}},
\end{aligned}
$$

where $\Gamma_{N_{1}}^{T}$ and $n_{N_{1}}^{\text {eq }}$ are the thermally averaged decay rate and the equilibrium abundance of the RH neutrinos, respectively. $\Gamma_{N_{1}}^{\mathrm{BH}}$ in Eq. (11b) is the decay width corrected by an average inverse time dilatation factor

$$
\Gamma_{N_{1}}^{\mathrm{BH}} \equiv\left\langle\frac{M_{N_{1}}}{E_{N_{1}}}\right\rangle_{\mathrm{BH}} \Gamma_{N_{1}}^{0} \approx \frac{\mathcal{K}_{1}\left(z_{\mathrm{BH}}\right)}{\mathcal{K}_{2}\left(z_{\mathrm{BH}}\right)} \Gamma_{N_{1}}^{0},
$$

where $\Gamma_{N_{1}}^{0}$ is the RH neutrino decay width, $\mathcal{K}_{1,2}(z)$ are modified Bessel functions of the second kind, and we defined $z_{\mathrm{BH}}=M_{N_{1}} / T_{\mathrm{BH}}$. The average is taken with respect to the $\mathrm{BH}$ instantaneous spectrum since the RH neutrino energies are distributed according to the Hawking rate, which resembles a thermal distribution. The approximated form of $\Gamma_{N_{1}}^{\mathrm{BH}}$ as a function of $\mathcal{K}_{1,2}(z)$ is obtained assuming that the Hawking spectrum has a Maxwell-Boltzmann form. In our numerical code we use the full graybody factors.

Equation (11b) contains a source term related to the $\mathrm{PBH}$ evaporation, equal to the $\mathrm{PBH}$ number density, $n_{\mathrm{BH}} \equiv$ $\varrho_{\mathrm{BH}} / M$, times $\Gamma_{\mathrm{BH} \rightarrow N_{1}}$, the $\mathrm{RH}$ neutrino emission rate

\footnotetext{
${ }^{2}$ We restrain from evolving with respect to the usual $z=$ $M_{N_{1}} / T$ parameter since the entropy is not conserved [54].
} 
per BH. To obtain $\Gamma_{\mathrm{BH} \rightarrow N_{1}}$, we integrate the Hawking rate, Eq. (3), with respect to the momentum [55-57],

$$
\begin{aligned}
\Gamma_{\mathrm{BH} \rightarrow N_{1}} & \equiv \int_{0}^{\infty} \frac{d^{2} \mathcal{N}_{N_{1}}}{d p d t} d p, \\
& \approx \frac{27 T_{\mathrm{BH}}}{32 \pi^{2}}\left(-z_{\mathrm{BH}} \mathrm{Li}_{2}\left(-e^{-z_{\mathrm{BH}}}\right)-\mathrm{Li}_{3}\left(-e^{-z_{\mathrm{BH}}}\right)\right),
\end{aligned}
$$

where the analytical approximation is obtained assuming the geometric optics limit, $\sigma_{\text {abs }}^{1 / 2}\left(M, M_{N_{i}}, p\right)=27 \pi G^{2} M^{2}$, being $\operatorname{Li}_{s}(z)$ are polylogarithm functions of order $s$.

The equation for the $B-L$ asymmetry, $n_{\alpha \beta}^{B-L}$, reads

$a H \frac{d n_{\alpha \beta}^{B-L}}{d a}=\epsilon_{\alpha \beta}^{(1)}\left[\left(n_{N_{1}}^{\mathrm{TH}}-n_{N_{1}}^{\mathrm{eq}}\right) \Gamma_{N_{1}}^{T}+n_{N_{1}}^{\mathrm{BH}} \Gamma_{N_{1}}^{\mathrm{BH}}\right]+\mathcal{W}_{\alpha \beta}$,

where $\epsilon_{\alpha \beta}^{(1)}$ is the $C P$-asymmetry matrix and $\mathcal{W}_{\alpha \beta}$ are the washout terms detailed in Appendix A. The generation of the lepton asymmetry has thermal and nonthermal sources stemming from the plasma and $\mathrm{PBH}$ evaporation respectively. However, the washout is independent of the PBHs.

Similar to the thermal leptogenesis scenario, the PBH induced leptogenesis can be understood through the $z_{\mathrm{BH}}$ parameter, cf. Eqs. (12) and (13). In the case that $z_{\mathrm{BH}} \gg 1$, the $\mathrm{RH}$ neutrino emission becomes suppressed as the $\mathrm{RH}$ neutrino mass exceeds the temperature of the black hole, and the effect on the final asymmetry is negligible. On the other hand, if $z_{\mathrm{BH}} \lesssim 1$, the PBHs emit $\mathrm{RH}$ neutrinos independently of the conditions of the surrounding thermal plasma. However, the final contribution to the baryon

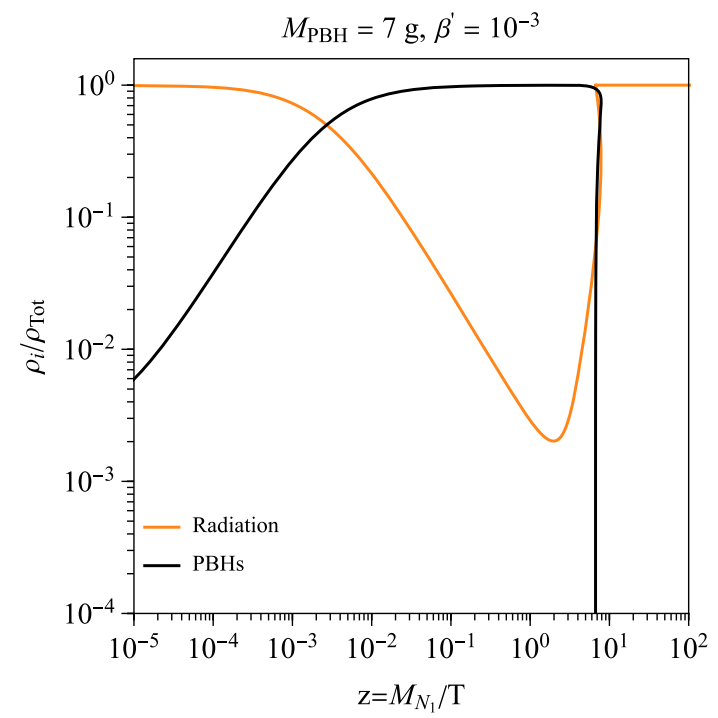

asymmetry is crucially dependent on the $C P$-asymmetry matrix $\left(\epsilon_{\alpha \beta}\right)$, related to the Yukawa couplings, and on the properties of the ambient plasma. Thus, according to the period when the black hole induced leptogenesis is active relative to the thermal leptogenesis era $\left(z=M_{N} / T \sim \mathcal{O}(1)\right)$, the PBH contribution can be substantial or not. For instance, if washout processes are ineffective when the RH neutrinos are emitted by the PBHs $(z \gtrsim 10)$, their contribution to the final baryon-to-photon ratio can be enhanced. Nevertheless, simultaneous to the RH neutrinos, PBHs produce a significant quantity of entropy, effectively reducing the baryon-to-photon ratio. This interplay will ultimately determine the final baryon asymmetry.

\section{RESULTS AND DISCUSSION}

We solve the system of equations (8), (10), (11), and (14), together with the PBH mass rate, Eq. (5), to obtain the final baryon asymmetry in a PBH dominated universe. For the sake of generality, we do not include any bound on the initial PBH fraction. We have implemented such system of equations in a plug-in to be used with the ULYSSES PYTHON package [32].

We present in Fig. 1 a prototypical solution for $M_{N_{1}}=10^{10} \mathrm{GeV}$ and Yukawa parameters as presented in Appendix B, while the $\mathrm{PBH}$ parameters are taken to be $M_{i}=7 \mathrm{~g}$ and $\beta^{\prime}=10^{-3}$. Other solutions are presented in the same App. B. The left panel of the aforementioned figure shows the ratio of the main Universe components (PBHs and radiation) to the total as function of the dimensionless parameter $z$. We observe that, for the chosen PBH values, a PBH domination occurs at about $z \sim 0.1$,

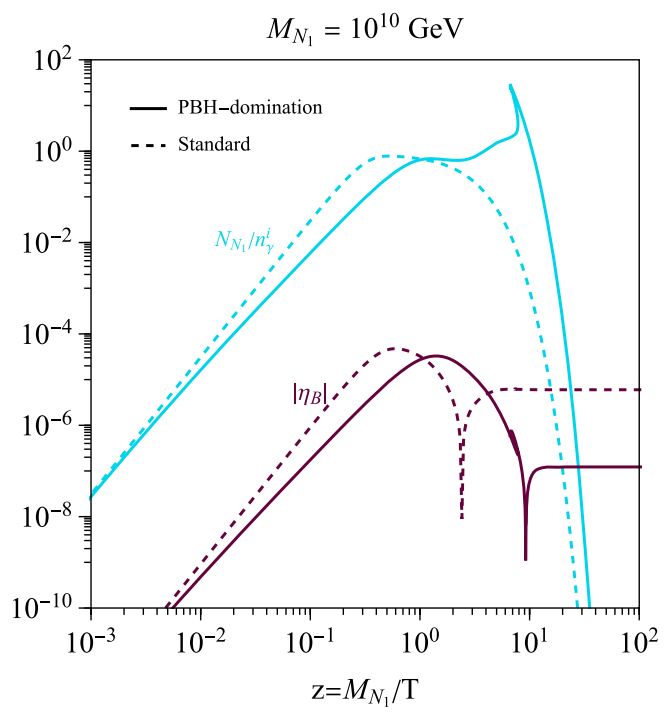

FIG. 1. An example solution of the full Boltzmann $+\mathrm{PBH}$ system of equations for $M_{i}=7 \mathrm{~g}, \beta^{\prime}=10^{-3}$, and $M_{N_{1}}=10^{10} \mathrm{GeV}$. The left plot shows the evolution of energy densities of the black holes (black) and radiation (orange) as a function of $z=M_{N_{1}} / T$. The right plot shows the evolution of the magnitude of right-handed neutrinos normalized to the initial photon density (blue) and the magnitude of the baryon-to-photon ratio (Tyrian purple) as a function of $z$, including PBHs (full lines) and the standard-without PBHs-scenario (dashed lines). 
close to the period where thermal leptogenesis takes place. As such, the RH neutrino and baryon-to-photon ratio are significantly modified due to the change in the evolution of the Universe. This is readily seen in the right panel of Fig. 1, where we present the RH neutrino density normalized to the initial photon density (blue) and $\eta_{B}$ (Tyrian purple) for the standard case-without $\mathrm{PBHs}$ - (dashed) and including the evaporation (full lines). In fact, when the PBH contribution becomes important $\left(z \sim 10^{-3}\right)$ the $\mathrm{RH}$ neutrino density starts to deviate from the standard case. Larger modifications to both $\mathrm{RH}$ neutrino density and baryon-to-photon ratio appear when the evaporation accelerates $(z \sim 1)$. The RH neutrino density increases because of the extra SM radiation coming from the evaporation, leading to additional scatterings that produce neutrinos. Simultaneously, the PBH evaporation creates RH neutrinos directly, although in a less significant manner than the plasma. Naively, we can imagine that the extra population of RH neutrinos should increase the lepton asymmetry. Nevertheless, the evaporation introduces a large amount of entropy, substantially reducing the asymmetry. For the specific case that we present, there is a reduction larger than one order of magnitude in the final value of $\eta_{B}$ (compare the dashed and full lines). Thus, we see that there exists a highly nontrivial interdependence between the lepton asymmetry generation and the $\mathrm{PBH}$ evaporation.

In Fig. 2 we present the baryon-to-photon ratio $\left|\eta_{\mathrm{B}}\right|$ as a function of the initial PBH mass $M_{i}$, for some specific sets of RH neutrino masses and Yukawa matrix elements. We assume $\beta^{\prime}=10^{-3}$ such that PBH domination is guaranteed. We will discuss the dependence on the initial fraction later on. The parameters related to the Yukawa matrix are provided in Appendix B and are chosen such that the baryon asymmetry will be maximally enhanced while preserving the perturbative series of the neutrino masses [41]. Therefore, the dilutionary effect we observe would only be more pronounced in other parameter space regions.

When the thermal leptogenesis era occurs before the PBH evaporation, we observe that the entropy injection largely dilutes the initial baryon-to-photon ratio. Depending on the $\mathrm{RH}$ neutrinos and initial $\mathrm{PBH}$ masses' specific values, the PBH induced asymmetry can partially mitigate the depletion of the final baryon-to-photon ratio. In the high mass regime $\left(10^{9} \mathrm{GeV} \lesssim M_{N_{1}} \lesssim 10^{12} \mathrm{GeV}\right.$, Fig 2, left panel), we observe the depletion of baryon asymmetry and the limited mitigation from the $\mathrm{PBH}$-induced leptogenesis depending on the initial PBH mass. Let us consider as benchmark the case of $M_{N_{1}}=10^{11} \mathrm{GeV}$ (red line). For $M_{i} \lesssim 1 \mathrm{~g}$, the final baryon-to-photon ratio is only marginally affected because the evaporation takes places before the thermal leptogenesis era. Thus, the RH neutrinos produced by the PBHs only constitute an initial condition for the leptogenesis. For $M_{i} \gtrsim 1 \mathrm{~g}$, the PBHs start to inject a large quantity of radiation, reducing significantly $\left|\eta_{\mathrm{B}}\right|$. However, for $1 \mathrm{~g} \lesssim M_{i} \lesssim 3 \times 10^{3} \mathrm{~g}$, the contribution coming from the RH neutrinos emitted by the PBHs ameliorate the diminution of the final baryon asymmetry. As such additional RH neutrinos are emitted after thermal leptogenesis, they do not experience strong washout effects. Therefore, as noted before, their out-of-equilibrium decays alleviate the reduction of the baryon asymmetry. This effect is maximal when $z_{\mathrm{BH}} \sim 1$, i.e.,

$$
M_{i} \sim \frac{1}{8 \pi G M_{N_{1}}} \sim 10^{2} \mathrm{~g}\left(\frac{10^{11} \mathrm{GeV}}{M_{N_{1}}}\right) .
$$

For PBH masses larger than such a value, the RH emission becomes suppressed, so that the only effect present

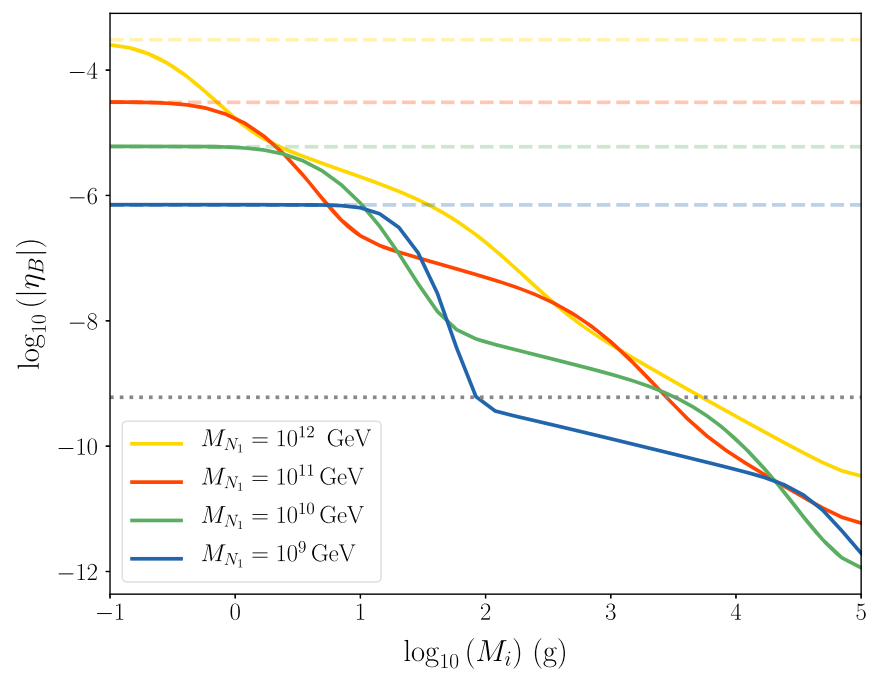

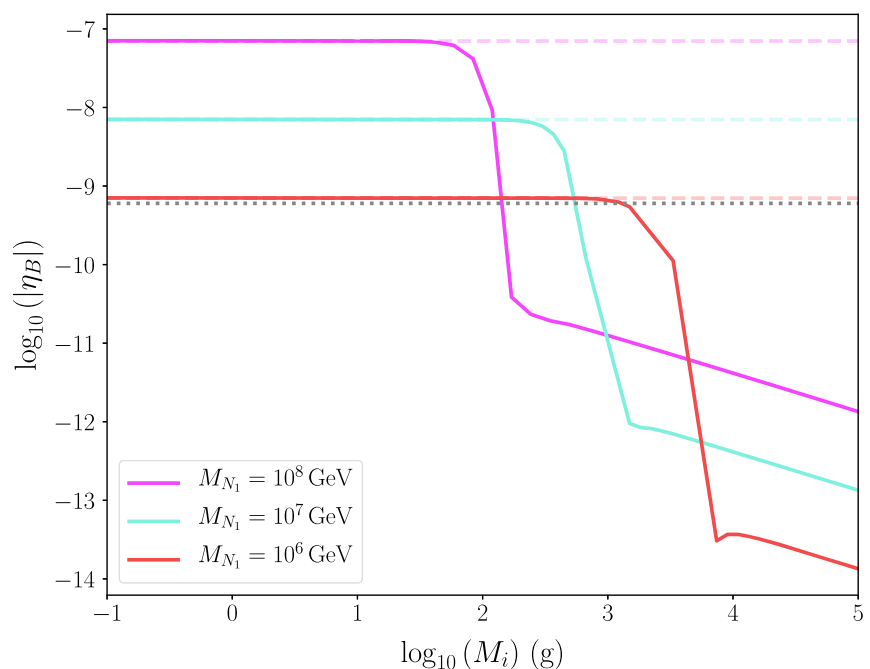

FIG. 2. The final baryon-to-photon ratio as a function of the initial black hole masses for varying masses of the RH neutrino. The grey dotted line shows the measured baryon-to-photon ratio [25] while the color dashed lines indicate the result obtained from leptogenesis in the case that there was no $\mathrm{PBH}$ dominated era. In both plots we assume $\beta^{\prime}=10^{-3}$. 
corresponds to the reheating of the primordial plasma. In fact, for $M_{i} \sim 10^{4} \mathrm{~g},\left|\eta_{\mathrm{B}}\right|$ is decreased by a factor of $\sim \mathcal{O}\left(10^{6}\right)$ with respect to minimal thermal leptogenesis scenario. For other values of the RH neutrino masses, similar behavior of $\left|\eta_{\mathrm{B}}\right|$ is observed. The partial mitigation due to the $\mathrm{PBH}-$ induced asymmetry is shifted in $M_{i}$ according to Eq. (15) and becomes less pronounced because the Yukawa couplings are smaller for lower RH neutrino masses, cf. Eq. (2). We emphasize that the points chosen here are highly fine tuned and significantly overproduce the baryon asymmetry in the absence of PBH entropy dilution. Therefore more generic points in the leptogenesis parameter space which produce just enough baryon asymmetry to be compatible with data would suffer the same dilution for $M_{i} \gtrsim 10^{4} \mathrm{~g}$ and be placed under tension. While the intermediate-mass regime is excluded for generic points in the model parameter space (for discussion see Ref. [58]) here we study fine-tuned points in order to draw general conclusions regarding entropy dilution from a PBH dominated early Universe. In this case, $10^{6} \mathrm{GeV} \lesssim M_{N_{1}} \lesssim 10^{9} \mathrm{GeV}$ (Fig 2, right panel), the injected entropy largely affects the total $\left|\eta_{\mathrm{B}}\right|$ for initial PBH masses $M_{i} \gtrsim 10^{2} \mathrm{~g}\left(3 \times 10^{3} \mathrm{~g}\right)$ in the case of RH neutrino masses $M_{N_{1}}=10^{8} \mathrm{GeV}\left(10^{6} \mathrm{GeV}\right)$. The mitigation present in the high-mass regime is absent here, as the contribution from PBH evaporation to the total $\left|\eta_{\mathrm{B}}\right|$ is reduced due to their smaller Yukawa couplings.

In Fig. 3, we present the dependence of the final baryon-to-photon ratio on the initial PBH fraction $\beta^{\prime}$ and mass $M_{i}$ for two benchmark cases of the RH neutrino parameters $-M_{N_{1}}=\left\{10^{8}, 10^{11}\right\} \mathrm{GeV}$. In the high mass regime, PBHs with masses $M_{i} \lesssim 1 \mathrm{~g}$ do not affect the final baryon asymmetry, independently of $\beta^{\prime}$, as noted previously. For $M_{i} \gtrsim 1 \mathrm{~g}$, we can distinguish two generic regions. If $\beta^{\prime} \gtrsim 10^{-4}$, we find a similar behavior as observed in Fig. 2: an increasing depletion of $\left|\eta_{\mathrm{B}}\right|$ for larger PBH masses, partially mitigated only for values in which $z_{\mathrm{BH}} \lesssim 1$. For lower values of $\beta^{\prime}$, the reduction caused by the entropy injection depends more on the initial PBH fraction. However, within the region leading to a $\mathrm{PBH}$ dominated era, the asymmetry is reduced by at least a factor of $\sim 5$. Similar features are present in the intermediate-mass regime benchmark. For $\beta^{\prime} \gtrsim 10^{-4}$, the depletion decreases the asymmetry at least by a factor of $\sim \mathcal{O}\left(10^{3}\right)$ for $M_{i} \gtrsim 10^{2} \mathrm{~g}$, independently of $\beta^{\prime}$. For smaller initial PBH fractions, the entropy injection diminishes the baryon-tophoton ratio by a factor between $\sim 5-10^{3}$ depending on the specific PBH initial fraction and mass. Nonetheless, let us stress that even the reduction by a factor of 5 induces a tension on the intermediate-mass regime.

\section{GRAVITATIONAL WAVE SIGNATURE}

From the recent advances in multi-messenger observations, we may wonder whether it is possible to demonstrate that existed an early PBH dominated era. Diverse studies have shown the possibility of testing leptogenesis and/or PBHs considering gravitational waves (GW) [59-63]. As an example, let us examine the stochastic background of GWs produced directly from the PBHs evaporation. Their energy density can be obtained by integrating the Hawking spectrum over the PBH lifetime,

$$
\begin{aligned}
\Omega_{\mathrm{GW}} h^{2}\left(f_{0}\right)= & \frac{8(2 \pi)^{4} f_{0}^{4}}{3 H_{0}^{2} M_{\mathrm{Pl}}^{6}} \frac{\varrho_{\mathrm{PBH}}^{i}}{M_{i}} \int_{1}^{a_{\mathrm{EV}}} \frac{d a}{a^{4} H} \\
& \times \frac{\sigma_{\mathrm{abs}}^{2}\left(2 \pi G M a_{0} f_{0} / a\right) M^{2}}{\exp \left[16 \pi^{2} G M a_{0} f_{0} / a\right]-1},
\end{aligned}
$$

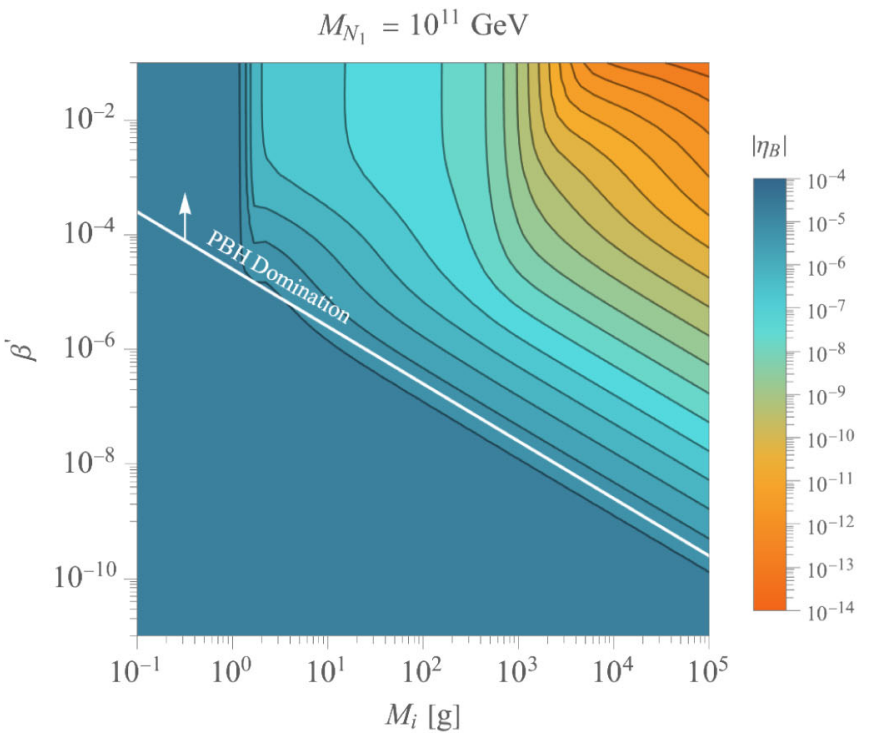

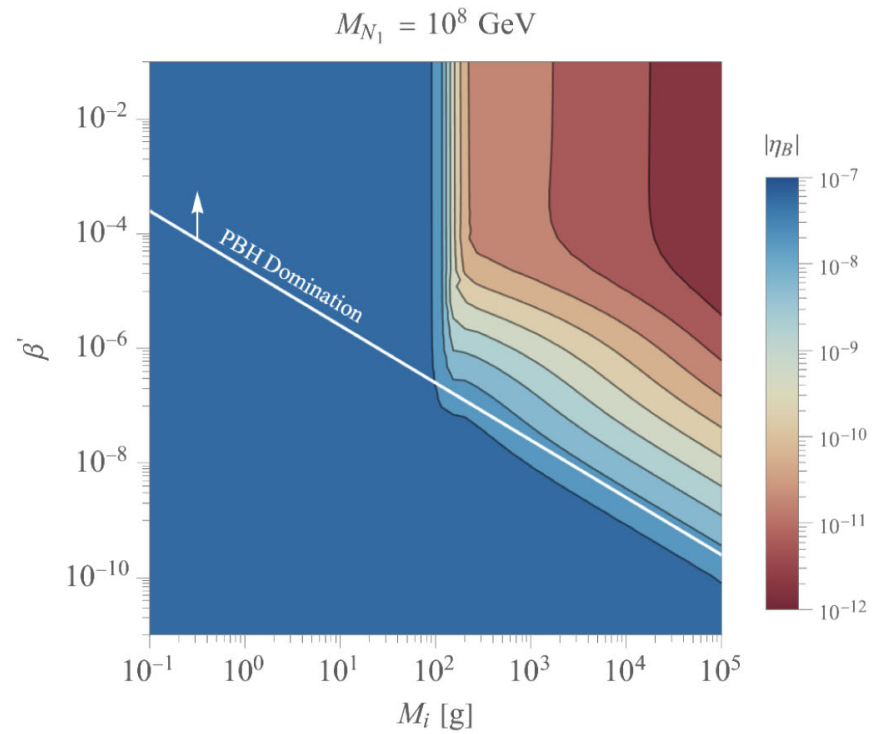

FIG. 3. $\left|\eta_{\mathrm{B}}\right|$ as function of the initial fraction $\beta^{\prime}$ and PBH mass $M_{i}$ for two different benchmarks, $M_{N_{1}}=10^{11} \mathrm{GeV}$ (left) and $M_{N_{1}}=10^{8} \mathrm{GeV}$ (right). The white line indicates the values from which it is expected an early PBH dominated era. 
being $M_{\mathrm{Pl}}=G^{-1 / 2}$ the Planck mass, $\frac{d^{2} \mathcal{N}_{g}}{d p d t}$ the Hawking emission rate for gravitons, $f_{0}$ the GW frequency and $a_{0}$ the scale factor today. Employing the solutions of Boltzmann Eqs., we present the GW spectrum for a range of PBH masses in Fig. 4 which are relevant for thermal leptogenesis. The peak frequency of the GW spectrum generated from evaporating $\mathrm{PBHs}$ is larger for higher initial masses. This shift occurs because the redshift effects are stronger for GW emitted from lighter PBHs, which evaporated earlier in the history of the universe. Therefore, we find that the GWs from Hawking emission have significantly high frequencies- $\mathcal{O}\left(f_{0}\right) \sim 100 \mathrm{THz}$ for $M_{i}=10^{-1} \mathrm{~g}$ - out of reach for current detectors. While detection of the GWs emitted by PBHs is challenging, there are some proposals [64-69], such as graviton-photon conversion [69], which aim to probe a high frequency regime, $f \sim \mathcal{O}(\mathrm{kHz}-\mathrm{MHz})$. So, perhaps in the future, there could exist a possible way to detect the extreme frequencies ( $f \gtrsim 10 \mathrm{THz}$ ) of the GWs from the evaporation.

Nonetheless, there may be other possible sources of GWs from leptogenesis combined with early PBH domination. First, it has been shown that phase transitions associated with the spontaneous symmetry breaking associated to the generation of right-handed neutrino masses can create GWs [60,70-72]. Second, if a nonmonochromatic mass spectrum of PBHs was considered then the inhomogeneity of the PBHs would create GWs in the frequency regime detectable by LISA [73,74]. These possibilities provide interesting avenues for further studies. Thus, we conclude that if evidence of a PBH dominated early universe consistent with initial PBH masses of $\mathcal{O}\left(M_{i}\right) \gtrsim 1 \mathrm{~kg}$ and $\beta^{\prime} \gtrsim 10^{-6}$ are found, this would place significant tension on the intermediate-scale leptogenesis scenario.

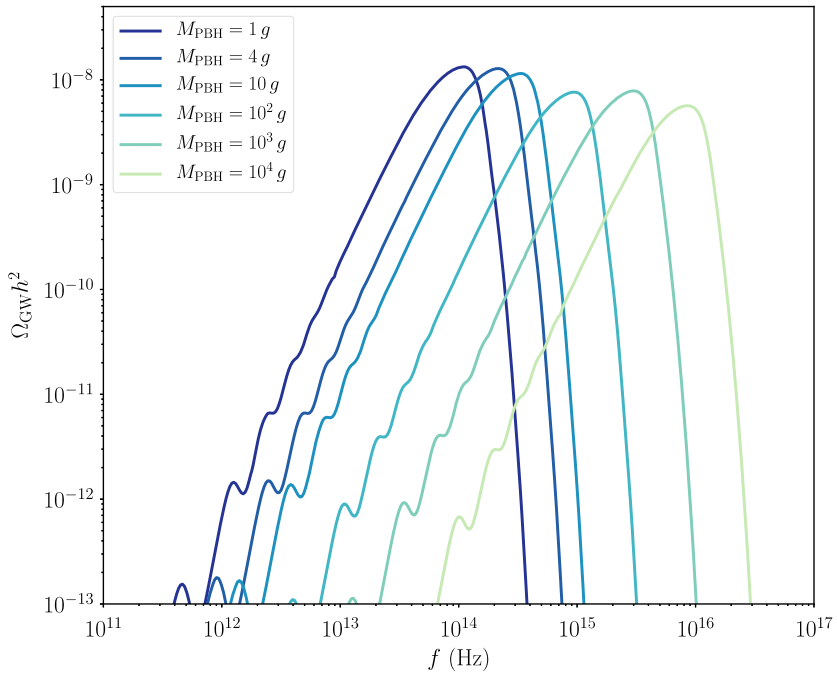

FIG. 4. Stochastic gravitational wave spectrum generated by evaporating primordial black holes.

\section{ACKNOWLEDGMENTS}

We want to thank Cecilia Lunardini for her involvement during the initial stages of this project. We are grateful to Kristian Moffat for useful discussions on leptogenesis. Fermilab is operated by the Fermi Research Alliance, LLC under Contract No. DE-AC02-07CH11359 with the United States Department of Energy.

Note added.-During the end stage preparation of this work, a paper by D. Hooper and G. Krnjaic appeared where they also consider primordial black hole induced leptogenesis [75].

\section{APPENDIX A: FRIEDMANN-BOLTZMANN EQUATIONS}

Since Friedmann equations for the evolution of the Universe cover a huge range in the scale factor $a$, in our code we evolve the system of equations with respect to the $\operatorname{logarithm}$ of $a, \alpha \equiv \log (a)$. Here, we provide explicitly the system of equations (5), (8), and (10) as function of $\alpha$

$$
\begin{aligned}
\frac{d M}{d \alpha} & =-\frac{\kappa}{H} \varepsilon(M)\left(\frac{1 \mathrm{~g}}{M}\right)^{2}, \\
\frac{d \varrho_{\mathrm{R}}}{d \alpha} & =-\frac{\varepsilon_{\mathrm{SM}}(M)}{\varepsilon(M)} \frac{1}{M} \frac{d M}{d \alpha} \varrho_{\mathrm{BH}}, \\
\frac{d \varrho_{\mathrm{BH}}}{d \alpha} & =\frac{1}{M} \frac{d M}{d \alpha} 10^{\alpha} \varrho_{\mathrm{BH}}, \\
\frac{d T}{d \alpha} & =-\frac{T}{\Delta}\left\{1+\frac{\varepsilon_{\mathrm{SM}}(M)}{\varepsilon_{\mathrm{D}}(M)} \frac{1}{M} \frac{d M}{d \alpha} \frac{g_{*}(T)}{g_{* S}(T)} \frac{10^{\alpha} \varrho_{\mathrm{PBH}}}{4 \varrho_{N}}\right\}, \\
H^{2} & =\frac{8 \pi G}{3}\left(\varrho_{\mathrm{BH}} 10^{-3 \alpha}+\varrho_{\mathrm{R}} 10^{-4 \alpha}\right) .
\end{aligned}
$$

The Boltzmann equations for the evolution of the $\mathrm{RH}$ neutrino number density and the lepton asymmetry in Eqs. (11), and (14) as function of $\alpha$, explicitly presenting the washout terms for the three flavor components, $e, \mu, \tau$, are given by

$$
\begin{aligned}
& \frac{d n_{N_{1}}^{\mathrm{TH}}}{d \alpha}=-\left(n_{N_{1}}^{\mathrm{TH}}-n_{N_{1}}^{\mathrm{eq}}\right) \frac{\Gamma_{N_{1}}^{T}}{H}, \\
& \frac{d n_{N_{1}}^{\mathrm{BH}}}{d \alpha}=-n_{N_{1}}^{\mathrm{BH}} \frac{\Gamma_{N_{1}}^{\mathrm{BH}}}{H}+\frac{\Gamma_{\mathrm{BH} \rightarrow N_{1}}}{H} \frac{\varrho_{\mathrm{BH}}}{M},
\end{aligned}
$$




$$
\begin{aligned}
\frac{d N_{\alpha \beta}^{B-L}}{d \alpha}= & \epsilon_{\alpha \beta}^{(1)}\left[\left(n_{N_{1}}^{\mathrm{TH}}-n_{N_{1}}^{\mathrm{eq}}\right) \frac{\Gamma_{N_{1}}^{T}}{H}+n_{N_{1}}^{\mathrm{BH}} \frac{\Gamma_{N_{1}}^{\mathrm{BH}}}{H}\right]-\frac{1}{2} \frac{W_{1}}{H}\left\{P^{0(1)}, N^{B-L}\right\}_{\alpha \beta} \\
& -\frac{\Gamma_{\mu}}{2 H}\left[\left(\begin{array}{lll}
1 & 0 & 0 \\
0 & 0 & 0 \\
0 & 0 & 0
\end{array}\right),\left[\left(\begin{array}{lll}
1 & 0 & 0 \\
0 & 0 & 0 \\
0 & 0 & 0
\end{array}\right), N^{B-L}\right]\right]_{\alpha \beta}-\frac{\Gamma_{\tau}}{2 H}\left[\left(\begin{array}{lll}
0 & 0 & 0 \\
0 & 1 & 0 \\
0 & 0 & 0
\end{array}\right),\left[\left(\begin{array}{lll}
0 & 0 & 0 \\
0 & 1 & 0 \\
0 & 0 & 0
\end{array}\right), N^{B-L}\right]\right]_{\alpha \beta},
\end{aligned}
$$

where $W_{1}$ is the washout related to the $N_{1}$ neutrino,

$$
W_{1}=\frac{1}{4} \Gamma_{N_{1}}^{T} \mathcal{K}_{2}(z) z^{2}
$$

and $P^{0(i)} \equiv c_{i \alpha} c_{i \beta}^{*}$ are projection matrices describing the washout of a specific flavor [76]. We solve this system of equations using ULYSSES PYTHON package [32]. However, we do not use the default conversion for the baryon-to-photon ratio (which assumes entropy conservation). Rather we normalize by the comoving number density of photons as outlined in our plugin. For a detailed discussion of Boltzmann equations which incorporates entropy production see Ref. [54].

\section{APPENDIX B: PROTOTYPICAL SOLUTIONS TO PBH-INDUCED LEPTOGENESIS}

Understanding the behavior of the $\mathrm{PBH}$-induced leptogenesis involves solving the system of coupled equations presented in (A1) and (A2). The enhancement and depletion of the baryon asymmetry, for differing PBHs and right-handed neutrino masses, cannot be captured with simple, closed analytic expressions. Here we show specifically the results for three benchmark points, labeled "before," "during," and "after" according to when the PBH disappearance occurs relative to thermal leptogenesis. We consider a region of the parameter space where the lepton asymmetry is enhanced due to fine-tuning of the R-matrix while retaining the perturbativity of the Yukawa couplings. The R-matrix has the following form,

$$
\begin{aligned}
R= & \left(\begin{array}{ccc}
1 & 0 & 0 \\
0 & c_{\omega_{1}} & s_{\omega_{1}} \\
0 & -s_{\omega_{1}} & c_{\omega_{1}}
\end{array}\right)\left(\begin{array}{ccc}
c_{\omega_{2}} & 0 & s_{\omega_{2}} \\
0 & 1 & 0 \\
-s_{\omega_{2}} & 0 & c_{\omega_{2}}
\end{array}\right) \\
& \times\left(\begin{array}{ccc}
c_{\omega_{3}} & s_{\omega_{3}} & 0 \\
-s_{\omega_{3}} & c_{\omega_{3}} & 0 \\
0 & 0 & 1
\end{array}\right),
\end{aligned}
$$

where $c_{\omega_{i}} \equiv \cos \omega_{i}, s_{\omega_{i}} \equiv \sin \omega_{i}$ and the complex angles are given by $\omega_{i} \equiv x_{i}+i y_{i}$ with $\left|x_{i}\right|,\left|y_{i}\right| \leq 180^{\circ}$ for $i=1,2,3$.

The parameters of the Yukawa matrix are determined by the Casas-Ibarra parametrization. We choose for this benchmark point the following values,
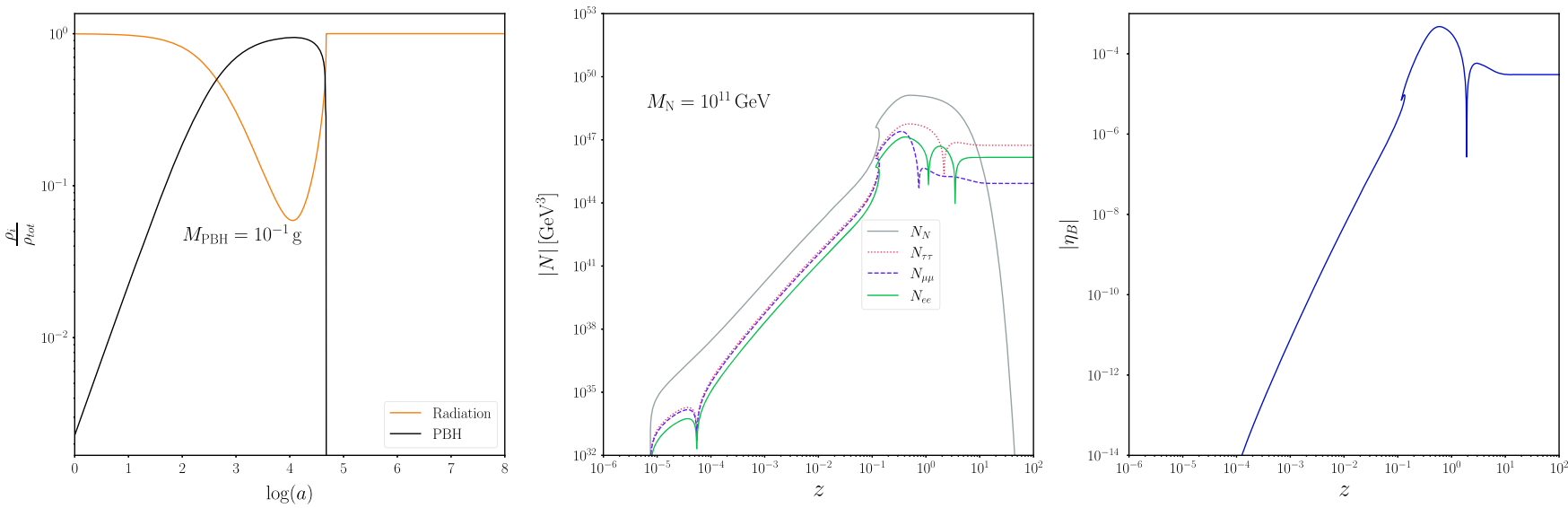

FIG. 5. The left plot shows the evolution of energy densities of the black holes (black) and radiation (yellow) as a function of the logarithm of the scale factor for the before scenario. The central plot shows the evolution of the magnitude of right-handed neutrino and flavored components of the lepton asymmetry comoving number densities as a function of the logarithm of $z=M_{N} / T$. The right panel presents the magnitude of the baryon-to-photon ratio as a function of the logarithm of $z$. 

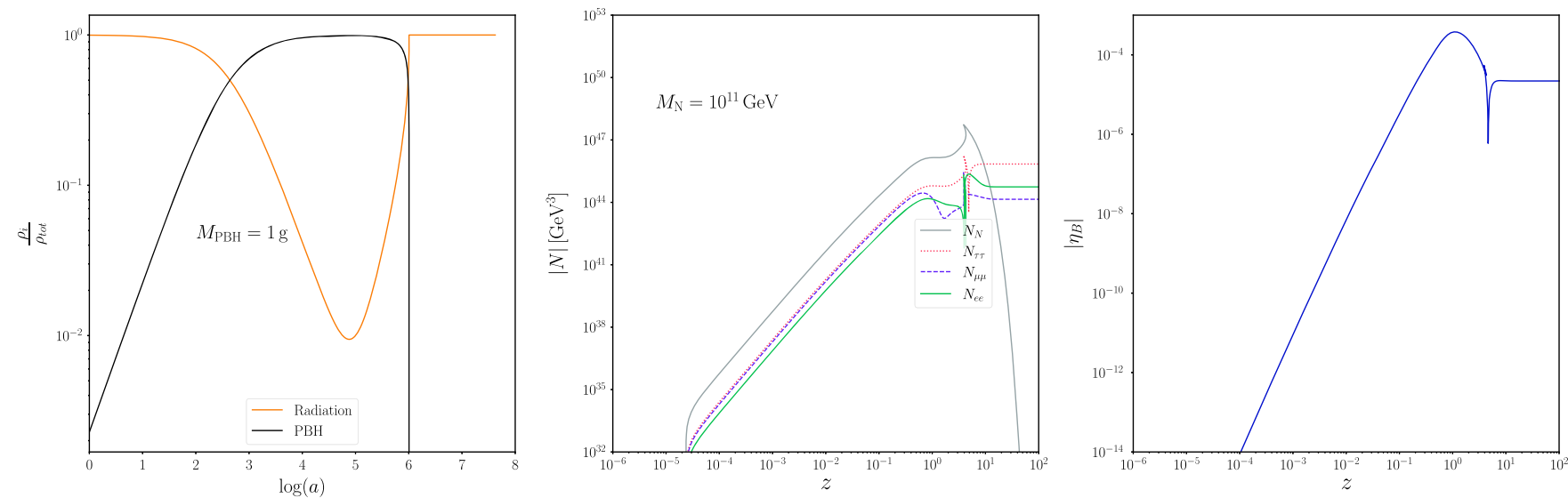

FIG. 6. The analogous plots for the during scenario.
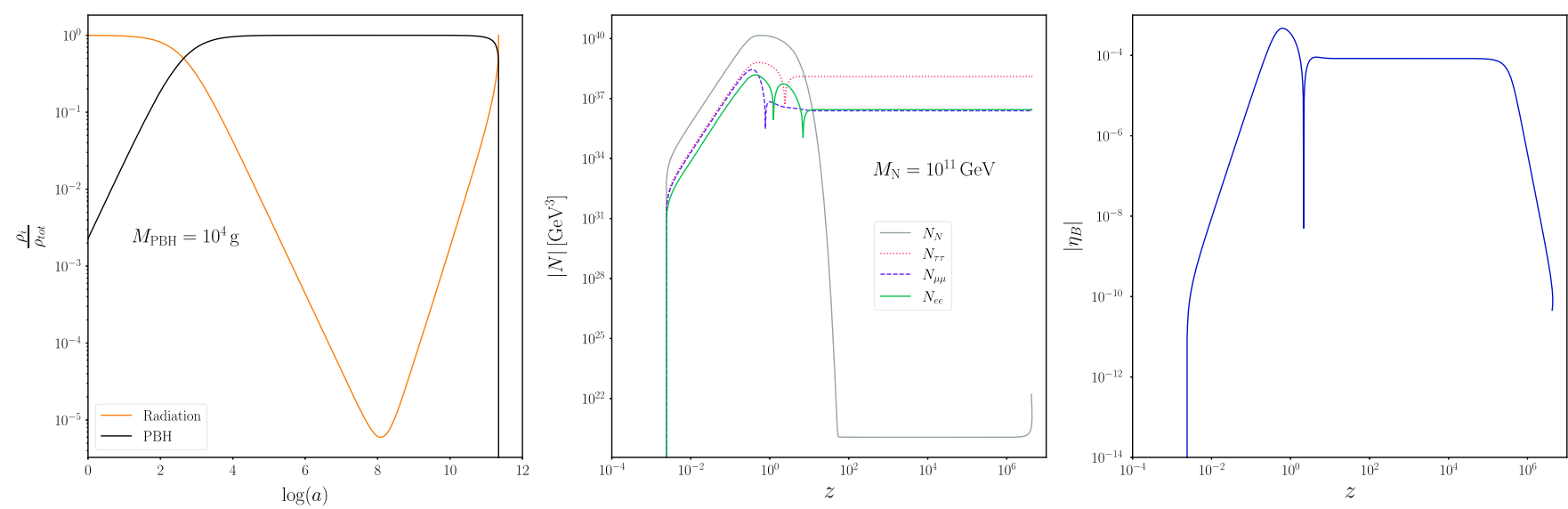

FIG. 7. The analogous plots for the after scenario.

$$
\begin{aligned}
m_{1} & =0.12 \mathrm{eV}, \quad M_{N_{1}}=10^{11} \mathrm{GeV}, \\
M_{N_{2}} & =3.16 \times 10^{11} \mathrm{GeV}, \quad M_{N_{3}}=10^{12} \mathrm{GeV}, \\
x_{1} & =132.2^{\circ}, \quad y_{1}=175.0^{\circ}, \quad x_{2}=87.8^{\circ}, \quad y_{2}=2.9^{\circ}, \\
x_{3} & =-30.3^{\circ}, \quad y_{3}=175.0^{\circ}, \quad \delta=281.2^{\circ}, \quad \alpha_{21}=181.90^{\circ}, \\
\alpha_{31} & =344.7^{\circ}, \quad \theta_{23}=46.2^{\circ}, \quad \theta_{12}=33.8^{\circ}, \quad \theta_{13}=8.61^{\circ},
\end{aligned}
$$

where we assume normal ordering and the masses of $m_{2}$ and $m_{3}$ are fixed from the best fit values of the mass squared splittings taken from [77]. The before, during, and after PBH masses are $10^{-1}, 1$ and $10^{4} \mathrm{~g}$ respectively. For each scenario, we take as the initial $\mathrm{PBH}$ fraction $\beta^{\prime}=10^{-3}$, such that it gives a $\mathrm{PBH}$ domination at some point in the evolution of the Universe.

Figure 5 shows the "before" scenario where the black hole disappearance occurs around $\log (a) \sim 4.5$. We observe the thermal plasma temperature raises slightly at this point (see lower left plot). The "time" when PBH-induced leptogenesis occurs corresponds to $z \sim 10^{-1}$, and we observe a spike in the comoving number density of the right-handed neutrinos in the upper right plot due to the nonthermal contribution from the PBHs. For $z>1$, PBH induced and thermal leptogenesis has completed, and the lepton asymmetry number density stabilizes. Figure 6 shows the during scenario where the black hole evaporation occurs around $\log (a) \sim 6$ and $z \sim 1$. Again, we observe a spike in the comoving number density of the right-handed neutrinos stemming from the explosive evaporation of the PBHs. This slight increase is not sufficient to overcome the washout processes which are still active in this regime, and the final baryon-to-photon ratio reduces slightly at $\log (a)>6$. Figure 7 shows the after scenario where the black hole evaporation occurs around $\log (a) \sim 11$ and $z \sim 10^{6}$. This is well after the era of thermal leptogenesis. We observe that the comoving number density of the right-handed neutrinos falls, due to Boltzmann 
suppression, until $\operatorname{large} \log (a)$ values when the PBHs populate them. In spite of this, there is a massive entropy dump by the $\mathrm{PBH}$, which produces photons thereby diluting the baryonto-photon ratio significantly.

\section{APPENDIX C: RH mass parameters}

Finally, we provide in Table I the RH neutrino masses used to obtain the baryon-to-photon ratio as function of the initial PBH mass, Fig. 1. The remaining parameters required by the Casas-Ibarra parametrization to obtain the Yukawa matrizes are the same given in Eq. (B2).
TABLE I. RH neutrino masses used to obtain the baryon-tophoton ratio, see main text.

\begin{tabular}{lcc}
\hline \hline$M_{N_{1}}$ & $M_{N_{2}}$ & $M_{N_{3}}$ \\
\hline $10^{6} \mathrm{GeV}$ & $3.16 \times 10^{6} \mathrm{GeV}$ & $10^{7} \mathrm{GeV}$ \\
$10^{7} \mathrm{GeV}$ & $3.16 \times 10^{7} \mathrm{GeV}$ & $10^{8} \mathrm{GeV}$ \\
$10^{8} \mathrm{GeV}$ & $3.16 \times 10^{8} \mathrm{GeV}$ & $10^{9} \mathrm{GeV}$ \\
$10^{9} \mathrm{GeV}$ & $3.16 \times 10^{9} \mathrm{GeV}$ & $10^{10} \mathrm{GeV}$ \\
$10^{10} \mathrm{GeV}$ & $3.16 \times 10^{10} \mathrm{GeV}$ & $10^{11} \mathrm{GeV}$ \\
$10^{11} \mathrm{GeV}$ & $3.16 \times 10^{11} \mathrm{GeV}$ & $10^{12} \mathrm{GeV}$ \\
$10^{12} \mathrm{GeV}$ & $3.16 \times 10^{12} \mathrm{GeV}$ & $10^{13} \mathrm{GeV}$ \\
\hline \hline
\end{tabular}

[1] B. Carr, K. Kohri, Y. Sendouda, and J. Yokoyama, arXiv: 2002.12778 .

[2] B. Carr and F. Kuhnel, Annu. Rev. Nucl. Part. Sci. 70, 355 (2020).

[3] M. Y. Khlopov, Res. Astron. Astrophys. 10, 495 (2010).

[4] S. W. Hawking, Nature (London) 248, 30 (1974).

[5] S. W. Hawking, Commun. Math. Phys. 43, 199 (1975).

[6] G. F. Chapline, Nature (London) 253, 251 (1975).

[7] J. H. MacGibbon, Nature (London) 329, 308 (1987).

[8] B. Carr, F. Kuhnel, and M. Sandstad, Phys. Rev. D 94, 083504 (2016).

[9] Y. Ali-Haimoud and M. Kamionkowski, Phys. Rev. D 95, 043534 (2017).

[10] B. J. Carr, K. Kohri, Y. Sendouda, and J. Yokoyama, Phys. Rev. D 81, 104019 (2010).

[11] S. Bird, I. Cholis, J. B. Munoz, Y. Ali-Haimoud, M. Kamionkowski, E. D. Kovetz, A. Raccanelli, and A. G. Riess, Phys. Rev. Lett. 116, 201301 (2016).

[12] K. Inomata, M. Kawasaki, K. Mukaida, Y. Tada, and T. T. Yanagida, Phys. Rev. D 96, 043504 (2017).

[13] L. Morrison, S. Profumo, and Y. Yu, J. Cosmol. Astropart. Phys. 05 (2019) 005.

[14] T. Fujita, M. Kawasaki, K. Harigaya, and R. Matsuda, Phys. Rev. D 89, 103501 (2014).

[15] O. Lennon, J. March-Russell, R. Petrossian-Byrne, and H. Tillim, J. Cosmol. Astropart. Phys. 04 (2018) 009.

[16] D. Hooper, G. Krnjaic, and S. D. McDermott, J. High Energy Phys. 08 (2019) 001.

[17] C. Lunardini and Y. F. Perez-Gonzalez, J. Cosmol. Astropart. Phys. 08 (2020) 014.

[18] D. Hooper, G. Krnjaic, J. March-Russell, S. D. McDermott, and R. Petrossian-Byrne, arXiv:2004.00618.

[19] I. Masina, Eur. Phys. J. Plus 135, 552 (2020).

[20] C. Keith, D. Hooper, N. Blinov, and S. D. McDermott, Phys. Rev. D 102, 103512 (2020).

[21] B. J. Carr, Astrophys. J. 206, 8 (1976).

[22] Ya. B. Zel'dovich, Pis'ma Zh. Eksp. Teor. Fiz. 24, 29 (1976) [JETP Lett. 24, 25 (1976)], http://jetpletters.ru/ps/1809/ article_27646.pdf.
[23] D. Baumann, P. J. Steinhardt, and N. Turok, arXiv:hep-th/ 0703250 .

[24] C. Patrignani et al. (Particle Data Group), Chin. Phys. C 40, 100001 (2016).

[25] P. A. R. Ade et al. (Planck Collaboration), Astron. Astrophys. 594, A13 (2016).

[26] S. Yu. Khlebnikov and M. E. Shaposhnikov, Nucl. Phys. B308, 885 (1988).

[27] M. Fukugita and T. Yanagida, Phys. Lett. B 174, 45 (1986).

[28] A. Pilaftsis, Phys. Rev. D 56, 5431 (1997).

[29] A. Pilaftsis and T. E. J. Underwood, Nucl. Phys. B692, 303 (2004).

[30] E. K. Akhmedov, V. A. Rubakov, and A. Yu. Smirnov, Phys. Rev. Lett. 81, 1359 (1998).

[31] T. Asaka and M. Shaposhnikov, Phys. Lett. B 620, 17 (2005).

[32] A. Granelli, K. Moffat, Y. Perez-Gonzalez, H. Schulz, and J. Turner, Comput. Phys. Commun. 262, 107813 (2021).

[33] R. N. Mohapatra and G. Senjanovic, Phys. Rev. Lett. 44, 912 (1980).

[34] M. Gell-Mann, P. Ramond, and R. Slansky, Conf. Proc. C 790927, 315 (1979), https://inspirehep.net/literature/9686.

[35] T. Yanagida, Conf. Proc. C 7902131, 95 (1979).

[36] P. Minkowski, Phys. Lett. 67B, 421 (1977).

[37] R. N. Mohapatra and G. Senjanovic, Phys. Rev. D 23, 165 (1981).

[38] M. Magg and C. Wetterich, Phys. Lett. 94B, 61 (1980).

[39] G. Lazarides, Q. Shafi, and C. Wetterich, Nucl. Phys. B181, 287 (1981).

[40] C. Wetterich, Nucl. Phys. B187, 343 (1981).

[41] K. Moffat, S. Pascoli, S. T. Petcov, H. Schulz, and J. Turner, Phys. Rev. D 98, 015036 (2018).

[42] K. Moffat, S. Pascoli, S. Petcov, and J. Turner, J. High Energy Phys. 03 (2019) 034.

[43] J. A. Casas and A. Ibarra, Nucl. Phys. B618, 171 (2001).

[44] N. D. Birrell and P.C.W. Davies, Quantum Fields in Curved Space, Cambridge Monographs on Mathematical Physics (Cambridge University Press, Cambridge, England, 1982).

[45] D. N. Page, Phys. Rev. D 13, 198 (1976).

[46] W. G. Unruh, Phys. Rev. D 14, 3251 (1976). 
[47] C. Doran, A. Lasenby, S. Dolan, and I. Hinder, Phys. Rev. D 71, 124020 (2005).

[48] J. H. MacGibbon and B. R. Webber, Phys. Rev. D 41, 3052 (1990).

[49] J. H. MacGibbon, Phys. Rev. D 44, 376 (1991).

[50] I. Baldes, Q. Decant, D. C. Hooper, and L. Lopez-Honorez, J. Cosmol. Astropart. Phys. 08 (2020) 045.

[51] F. Hahn-Woernle, M. Plumacher, and Y. Y. Y. Wong, J. Cosmol. Astropart. Phys. 08 (2009) 028.

[52] N. Bernal and F. Hajkarim, Phys. Rev. D 100, 063502 (2019).

[53] P. Arias, N. Bernal, A. Herrera, and C. Maldonado, J. Cosmol. Astropart. Phys. 10 (2019) 047.

[54] W. Buchmuller, K. Schmitz, and G. Vertongen, Nucl. Phys. B851, 481 (2011).

[55] D. N. Page, Phys. Rev. D 16, 2402 (1977).

[56] D. N. Page, B. J. Carr, and J. H. MacGibbon, Phys. Rev. D 78, 064044 (2008).

[57] J. H. MacGibbon, B. J. Carr, and D. N. Page, Phys. Rev. D 78, 064043 (2008).

[58] W. Buchmuller, P. Di Bari, and M. Plumacher, Ann. Phys. (Amsterdam) 315, 305 (2005).

[59] A. D. Dolgov and D. Ejlli, Phys. Rev. D 84, 024028 (2011).

[60] J. A. Dror, T. Hiramatsu, K. Kohri, H. Murayama, and G. White, Phys. Rev. Lett. 124, 041804 (2020).

[61] S. Blasi, V. Brdar, and K. Schmitz, Phys. Rev. Research 2, 043321 (2020).

[62] R. Samanta and S. Datta, J. High Energy Phys. 05 (2021) 211.
[63] K. Inomata, M. Kawasaki, K. Mukaida, T. Terada, and T. T. Yanagida, Phys. Rev. D 101, 123533 (2020).

[64] A. Arvanitaki and A. A. Geraci, Phys. Rev. Lett. 110, 071105 (2013).

[65] A. Ito, T. Ikeda, K. Miuchi, and J. Soda, Eur. Phys. J. C 80, 179 (2020).

[66] Y. Chen, M. Kadic, D. E. Kaplan, S. Rajendran, A. O. Sushkov, and M. Wegener, arXiv:2007.07974.

[67] A. S. Chou et al. (Holometer Collaboration), Phys. Rev. Lett. 117, 111102 (2016).

[68] M. A. Page et al., arXiv:2007.08766.

[69] A. Ejlli, D. Ejlli, A. M. Cruise, G. Pisano, and H. Grote, Eur. Phys. J. C 79, 1032 (2019).

[70] V. Brdar, A. J. Helmboldt, and J. Kubo, J. Cosmol. Astropart. Phys. 02 (2019) 021.

[71] T. Hasegawa, N. Okada, and O. Seto, Phys. Rev. D 99, 095039 (2019).

[72] P. Di Bari, D. Marfatia, and Y.-L. Zhou, arXiv:2106.00025.

[73] T. Papanikolaou, V. Vennin, and D. Langlois, J. Cosmol. Astropart. Phys. 03 (2021) 053.

[74] G. Domènech, C. Lin, and M. Sasaki, J. Cosmol. Astropart. Phys. 04 (2021) 062.

[75] D. Hooper and G. Krnjaic, Phys. Rev. D 103, 043504 (2021).

[76] S. Blanchet, P. Di Bari, D. A. Jones, and L. Marzola, J. Cosmol. Astropart. Phys. 01 (2013) 041.

[77] I. Esteban, M. Gonzalez-Garcia, M. Maltoni, T. Schwetz, and A. Zhou, J. High Energy Phys. 09 (2020) 178. 